\title{
Concept of Dirty Dozen: The Silent Killers of Human Factor Errors and Mistakes
}

\author{
Ahasan $\mathrm{R}^{1 *}$, Shahren $\mathrm{AZA}^{2}$, Haque $\mathrm{W}^{3}$ and Islam $\mathrm{E}^{3}$ \\ 1Department of Industrial Engineering and Management, University of Oulu, Finland \\ ${ }^{2}$ Department of Cognitive Sciences, University Malaysia Sarawak, Malaysia \\ ${ }^{3}$ Faculty of Social Sciences, University Sultan Zainal Abidin, Kuala Terengganu, \\ Malaysia
}

\section{Review Article \\ Volume 2 Issue 1}

Received Date: December 29, 2017

Published Date: January 31, 2018

DOI: $10.23880 /$ eoij-16000137

*Corresponding author: Rabiul Ahasan, Department of Industrial Engineering and Management, University of Oulu, Finland, Tel: +6096662815; Email: rabiulahasan123@gmail.com

\section{Abstract}

Human Factors (HFs) interactions are important that surely impact the employee behavior at work. HFs interactions that interact with individual worker behavior can complement unintentional consequences at work. Currently, these consequences at work are being seen as the notorious culprits of HFs. Safety policy and systematic procedures of industries and companies seems to be strongly okay, however, dirty dozen concepts are not emphasized in workplace health and safety program. Dirty dozen is the twelve HFs problems that cause human errors or operator mistakes. In many project team applications, these are potential human causal factors that degrade worker skills and abilities. Dirty dozen are the real killers in every workplace environment which are also hidden behind the work process. Hence, this paper explores the basic concepts behind the dirty dozen, the silent killers of HFs errors, which are blamed for degrading worker abilities to perform job-tasks effectively and safely.

Keywords: Dirty Dozen; Human Factor; Errors and Mistakes; Resource Management

\section{Introduction}

Importance of human factors (HFs) interactions with day to day working life has been unexplored. HFs interactions in the work system open new research questions, especially on the concept of dirty dozen [1,2]. Hence, we need to explore the correct answers on the negative interaction issues (e.g., dirty dozen) that affect human abilities and work performances. The dirty dozen is a list of negative HFs concerns and issues hidden at various workplaces which may lead to severe consequences. Dirty dozen are the hidden killers, commonly found in the work dynamics of job-tasks, in every industries and companies. The dirty dozen are the twelve common causes [3,4], identified several years ago by the Canadian ergonomists (https://aceergocanada.ca/resources/magazine/index.html).

The concept of dirty dozen is applicable in every part of everyone's working life from lorry drivers to medical doctors. These factors affect the operator, the pilot, the mechanic, aircraft engineer, office secretary, and even the vehicle driver. The first one is the lack of communication where clear or direct statements can be missing. Without direct communication, it is critical to conduct safe operation in a see-and-be-seen environment $[5,6]$. The 


\section{Ergonomics International Journal}

second one is complacency that means someone's skills and experience exudes over estimation or due to over confidence. Complacency sometimes descends upon unawareness or lack of alertness against the impending danger at work. Self-satisfaction accompanied by unawareness of the potential dangers at work is a strong hedge against complacency. The third one is lack of knowledge or correct information in the job-task at hand. It means technical or non-technical information, checklist, or safety procedures may not adequately be translated correctly, or available in local language to the operation and maintenance team, for example. The fourth one is distraction (e.g., drawing one's attention away from the job-tasks). A distraction is anything that takes our mind off the task at hand. Distractions can cause us to think that we are further ahead in the work processes, or the systems than we are now. The fifth one is lack of teamwork to achieve a common goal. The sixth one is fatigue (e.g., decreased level of consciousness) that can cause weariness, exertion, nervousness and exhaustion. The seventh one is lack of resources. It means failure to use or acquire appropriate tools, equipment, information and procedures. In this case, we must not improvise when it comes to using the correct tools, manuals, or instructions. The next one is work pressure that creates a sense of urgency. Work pressure is also caused by lack of planning or execution of job-tasks on our part. In this situation, we should not over-promise and under-deliver anything related to the job-tasks. The ninth one is lack of assertiveness (e.g., lack of positive communication of needs), which is important for a new work system or process. Questions and doubts on the reliability or factuality of data and information can be a part of assertiveness. The tenth one is work-related stress that can be self-induced, and it has been caused by an outside sources. The eleventh one is lack of awareness, considered as the failure to be alert or vigilant in observing while working. The last one is norms, the way things are normally done. Norm is commonly accepted practices where assumptions are made without revalidating or verifying the current systems or procedures. Norms may have been formed differently based on different working culture. Norm is something that nothing has happened to sudden changes since local employees wish to stay in place. Unfortunately, in most cases, negative type norms are changed by accident, and not before. Therefore, industries and companies must putsafety first in their work system to minimise negative effects of dirty dozen by integrating HFs principles in employee training and education. Continuous and ongoing training and education can help us in reminding those casual factors or dirty dozen [7,8]. Alertness, friendly communication, resource management and safety precautions will definitely reduce HFs related errors and mistakes at work. Every time, employees should keep themselves alert and remind themselves against the negative consequences of dirty dozen. Every employee should look out and prevent themselves or other coworkers from being victims of HFs related mistakes and errors.

\section{Human Factors Related Errors and Mistakes}

About $80 \%$ of maintenance mistakes involve human factors (HFs) related errors in the aviation industry $[9,10]$. Errors and mistakes $[11,12]$ can also happen due to lack of on-the-job training. Studies [13-15] showed that errors and mistakes are involved in operation and maintenance type of tasks. If such work-related errors and mistakes $[16,17]$ are not detected then those will kill us by causing incidents, accidents, and deaths. An error is a mistake, the state or occurrences of wrong doing. Errors and mistakes can further be categorized into unintentional and intentional. An unintentional error is unintended that can deviate us from an accuracy or compete the perfect tasks. It is obvious that the split-line bolts were under-torque, for example, whether it was intentional or unintentional. Maybe we ignore the out-ofcalibration torque wrench for whatever reasons. Undertorque the same set of split-line nuts and bolts can cause serious accidents in an aviation industry. Unintended errors and mistakes are hidden or disguised even the management team may have a strong safety culture in the company.

A new system or operating an imported system or technology can be unfamiliar to many of the local employees in a local company. In many cases, the experienced team members may do mistakes in their decision of doing things improperly. Sometimes, the project teams have the tendency to accept technical or operating manuals given by the management those may not be right all the time. The correct way of doing something may not always be easy. Sometimes errors and mistakes are unavoidable even the employees follow standard work procedures at work. An experienced project team may have safety skills but errors and mistakes are unavoidable. The experienced and qualified personnel also commit simple error or mistake. Hidden unsafe nets in the work system hinder an established process or procedure. It can also lead to devastating injuries, accidents, and deaths $[18,19]$.

HFs programs, procedures, and checklists may work well on paper. But in reality, it is the hands on the notorious dirty dozen, the hidden killers at workplace. There are many unfortunate events at work due to the 
casual effects of HFs, the dirty dozen, those drives the loss of operation and create problems in operation, maintenance, logistic and other work processes. Production loss also happens due to a lack of safety procedures available in the plant, and bad ergonomics at workplace. Non-compliances of HFs guidelines and poor ergonomics are also blamed for increasing errors and mistakes. We therefore should not be complacent on the issues, concern, or occurrence of dirty dozen at any costs. In advance, we must know the impending problems at work. We must be very careful of potential hazards of dirty dozen. If we know our errors and mistakes, then we will not be imperilled in a dangerous situation.

\section{Avoid the Dirty Dozen 12 Common Causes of Human Factors Errors}

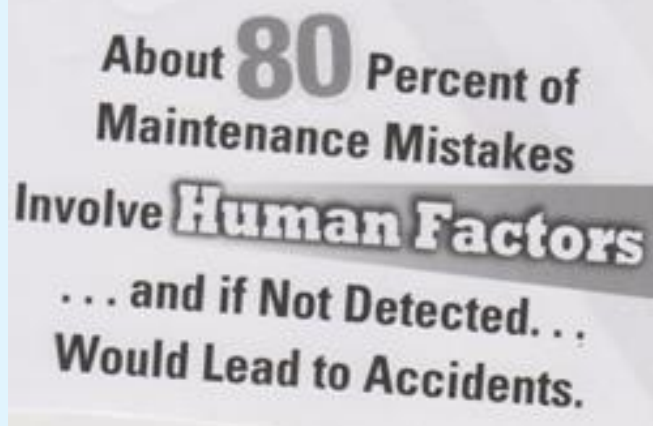

Figure 1: Dirty Dozen Placard.

\section{Discussion and Conclusion}

Dirty dozen are the twelve HFs problems; those are being seen as notorious culprits of human errors or mistakes. Therefore, dirty dozen issues have to be dealt with cautiously and seriously. Proper education, knowledge and training should continue against the bad effects of dirty dozen. HFs principles and ergonomic guidelines [20,21] should be incorporated in operation and maintenance type of work. Maintenance team should use the dirty dozen concepts during their daily work cycles for preventing work-related risk factors. The dirty dozen concepts should also be highlighted in media (e.g., tv-news, periodicals and daily newspapers), especially on the lack of situational awareness, complacency, and ill effects of miscommunication. Extra effort is also needed to link the lessons learnt back to our day-to-day tasking. Appropriate computer support or robotics can be useful for solving complicated work systems. Continuous training and education allow safety officers to understand the HFs causal factors (dirty dozen) in line with error-free operation and maintenance $[22,23]$. We need to understand human factors interactions to examine the relationship between the work systems, and existing work culture [24-26].

HFs are the factors at work with which a human worker interact with various challenges. HFs elements at work include the job-tasks itself; individual worker or employee behaviour. How a human worker behaves or act to a particular work environment is considered as HFs. A failure to apply HFs is also the key aspect of dirt dozens (e.g., adverse events at work). We must always be alert to avoid the ill consequences and negative effects of dirty dozen. For this, HFs policies, procedures and recommendations must be followed to minimise mistakes and remove errors and mistakes from all the workplaces. Individual's behavioural response within the job-task and work system must follow those checklists, guidelines. Integrating HFs guidelines, researchers [27-30] also illustrated recommendations for reducing errors and mistakes at work. It is thus important to keep good work performances through the safe work procedure and practices. HFs interactions with those twelve common mistakes can be used as a safety mechanism. However, the past incidents and accidents are the best to learn for future action [31]. Dirty dozen concepts should be used as important tools to identify, analyse, investigate, and internalize HFs related causal factors. Further studies and research should be conducted for deeper understanding of HFs interactions with the dirty dozen.

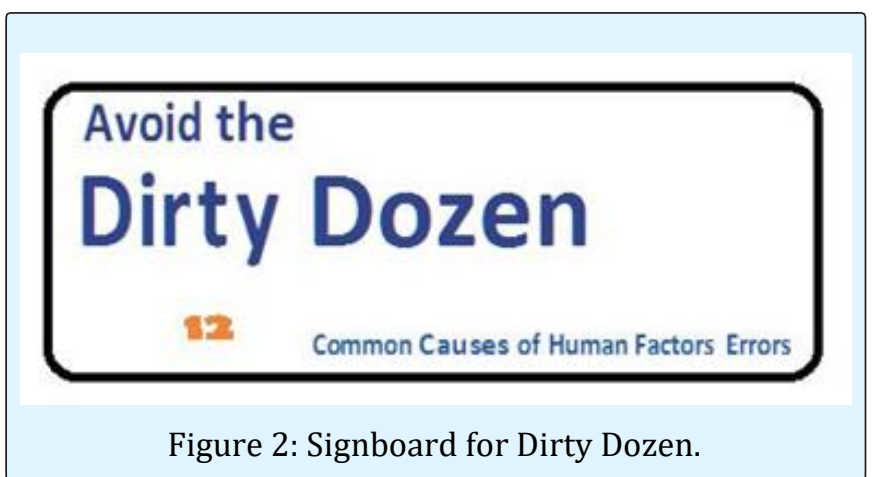

\section{Acknowledgement}

There were no funds or research grants available to support this research. The authors wrote this paper themselves without any third party involvement. 


\section{Ergonomics International Journal}

Appreciations are given to the anonymous reviewers for their critics and comments. There is no conflict of interest with anyone, including other co-authors.

\section{References}

1. Tolleson T (2016) Human Factors of Dirty Dozen: the curse of complacency. Blue Tuna Training and Documentation in Rockwall, Texas, USA.

2. Siong AK (2012) The dirty dozen. Air Force Inspectorate, HQ RSAF (Singapore), FOCUS 70: 1-30.

3. Common Causes of Human Factors Errors (2017). Available https://www.faasafety.gov/files/gslac/library/docu ments/2012/Nov/71574/DirtyDozenWeb3.pdf〉, Retrieved on 31/12/2017.

4. YOUR SOURCE FOR AVIATION SAFETY. Available at http://www.FAASafety.gov, Retrieved on 31/12/2018. Mech Spring (2002) Naval Safety Centre.

5. MechSpring (2002). Available at http://www.safetycentre.navy.mil/media/mech/ issues/spring02/human facors.htm, Retrieved 30 June, 2016.

6. Charlotte A (2010) Human Factors: beyond the dirty dozen.

7. Charlotte A (2010). Human Factors: beyond the dirty dozen. Available at http://www.maintenanceworld.com/human-factorsbeyond-the-dirty-dozen/, Accessed on 30/6/2016.

8. Steber D (2002) Human factors in maintenance: working to reduce error.

9. Wiegmann DA, Shappell SA (2000) A human error approach to aviation accident analysis: The human factors analysis and classification system. Office of Aviation Medicine, Washington DC, USA.

10. Kohn LT, Corrigan JM, Donaldson MS (1999) To err is human-building a safer health system. Committee on quality of health care in America. Institute of Medicine, National Academy Press, USA.

11. UKCAA (1992) Maintenance Error. Asia Pacific Air Safety.

12. Hobbs A, Williamson A (1998) Aircraft Maintenance Safety Survey-Results. Department of Transport and
Regional Services. Australian Transport Safety Bureau 43.

13. Goglia J (2000) Unpublished Statement. In: Proceedings of the $14^{\text {th }}$ Human Factors in Aviation Maintenance Symposium. Advances in Aviation Safety Conference, Vancouver.

14. John K (1999) Human factors in maintenance presentation preview. Naval Safety Centre.

15. John K (1999). Human factors in maintenance presentation preview. Naval Safety Centre. Available

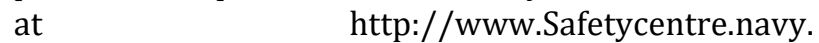
mil/presentations/aviation/hfacsmaintenance.htm, Retrieved 30 June, 2016.

16. Byrd J (2013). Human factors in aircraft maintenance. Available at http://www.gwbaa.com/resources/documents/stan ddown-byrd.pdf, Retrieved on 31/12/2017.

17. Dawson D, Reid K (1997) Fatigue, alcohol and performance impairment. Nature 388 (6639): 235237.

18. Accident Prevention Strategies (1993) Commercial Jet Aircraft Accidents World Wide Operations 19821991.

19. Joint Aviation Authorities (2001) Human factors in maintenance working group report: JAA maintenance human factors working group.

20. System Safety Services (2000) History of human factors training for aircraft maintenance personnel.

21. UK-HFCAG (1999) People, practices and procedures in aviation engineering and maintenance. Sample Staff Opinion Survey, pp: 20-23.

22. DuPont Sustainable Solutions (2016) HFs in safety and operations-how to change instinctive and habitual at risk behaviour.

23. (2017) Personal Development Training with Sid Savara. 13 ways to improve your concentration with a distraction free work environment.

24. AMT Handbook (Chapter-14).

25. AMT Handbook (Chapter-14). Available at https://www.faa.gov/regulations_policies/handbook s_manuals/aircraft/media/AMT_Handbook_Addendu m_Human_Factors.pdf, Retrieved on 31/12/2017. 


\section{Ergonomics International Journal}

26. Shouhed D, Gewertz B, Wiegmann D, Catchpole K (2012) Integrating human factors research and surgery: a review. Archives of Surgery 147(12): 11411146.

27. Harry SH (2004) Criteria for performance excellence. Baldridge national quality program. National Institute of Standards and Technology. Technology Administration. Department of Commerce, USA.

28. Grabe RC, Marx DA (1993) Reducing human error in aviation maintenance operations. In: Proceedings of the $46^{\text {th }}$ Annual International Air Safety Seminar, Flight Safety Foundation, Malaysia.

29. Byrnes WA, Black RE (1993) Developing and implementing CRM programs. In: Weiner E, Kanki B, Helmreich R (Eds.), Cockpit Resource Management. Academic Press, San Diego, USA.

30. King D (1998) Learning lessons the (not so) hard way. Incidents-the route to human factors in engineering. In: Proceedings of the $12^{\text {th }}$ Symposium on Human Factors in Aviation maintenance. 